IJMMS 25:4 (2001) 273-287

PII. S0161171201004306

http://ijmms.hindawi.com

(C) Hindawi Publishing Corp.

\title{
TOPOLOGICAL DEGREE AND APPLICATION TO A PARABOLIC VARIATIONAL INEQUALITY PROBLEM
}

\author{
A. ADDOU and B. MERMRI
}

(Received 3 January 2000)

\begin{abstract}
We are interested in constructing a topological degree for operators of the form $F=L+A+S$, where $L$ is a linear densely defined maximal monotone map, $A$ is a bounded maximal monotone operators, and $S$ is a bounded demicontinuous map of class $\left(S_{+}\right)$with respect to the domain of $L$. By means of this topological degree we prove an existence result that will be applied to give a new formulation of a parabolic variational inequality problem.
\end{abstract}

2000 Mathematics Subject Classification. Primary 47H11, 35K30, 47H05.

1. Introduction. The subject to which this paper is devoted, topological degree, is one of the most useful tools to study the existence of solutions of nonlinear problems. This tool has a powerful property known as the invariance under homotopy, which assures under appropriate hypotheses that the solvability of family of problems is invariant under continuous perturbations.

The notion of topological degree was first introduced explicitly by Brouwer (cf. [7]) in 1912 for continuous mappings from a bounded subset of $\mathbb{R}^{n}$ to $\mathbb{R}^{n}$.

In 1934, Leray and Schauder extended this concept to infinite dimensional Banach spaces with mappings of the form $f=I-g$, where $I$ is the identity map and $g$ is a compact map (see [7]). The uniqueness of the two degree functions was proved independently by Fuhrer in 1972 and Amann and Weiss [1], respectively. Browder [5, 6] extended the concept of this theory for operators of monotone type from a reflexive Banach space $X$ to its dual space $X^{*}$, mapping of class $\left(S_{+}\right)$, perturbation of maximal monotone operators by mappings of class $\left(S_{+}\right)$, and other special cases defined on Sobolev spaces. A new and easy construction of the topological degree for mappings of class $\left(S_{+}\right)$from separable Banach space to its dual space, is given by Berkovits and Mustonen [3]. In [4] they constructed a degree function for a class of mappings of the form $F=L+S$, where $L$ is a linear densely defined maximal monotone map from the domain $D(L)$ in $X$ to $X^{*}$ and $S$ is a bounded demicontinuous map of class $\left(S_{+}\right)$[4].

We construct a topological degree for operators of the form $L+A+S$ from $D(L) \subset X$, domain of $L$, to $2^{X^{*}}$, where $A$ is a bounded maximal monotone operator, $L$ is a linear densely defined maximal monotone map, and $S$ is a bounded demicontinuous map of class $\left(S_{+}\right)$with respect to $D(L)$. For the construction, we use the method used by Berkovits and Mustonen [4] and Yosida approximations to give a family of mappings of class $\left(S_{+}\right)$with respect to $D(L)$, where the $\left(S_{+}\right)$-degree (see [5]) is well defined on the reflexive Banach space $Y=D(L)$ equipped with the graph norm. By means of this topological degree function, we prove an existence result that will be applied to give 
a new formulation of the following parabolic variational inequality problem:

$$
\left(P_{\mathscr{K}}\right)\left\{\begin{array}{l}
\text { find } u \in D(L) \subset X \text { such that } \\
\int_{0}^{T}\left\langle\frac{\partial u(t)}{\partial t}, v(t)-u(t)\right\rangle+\left\langle-\Delta_{p}(u), v-u\right\rangle+(h, v-u) \geq 0, \quad \forall v \in \mathscr{K},
\end{array}\right.
$$

where

$$
\begin{aligned}
& \mathscr{K}=\left\{v \in L^{p}\left(0, T, W_{0}^{1, p}(\Omega)\right): v(t) \in K\right\}, \\
& K=\left\{v \in W_{0}^{1, p}(\Omega): v \geq 0 \text { a.c. in } \Omega\right\},
\end{aligned}
$$

and $h$ is an element of $L^{p^{\prime}}(Q)$ with $\left.Q=\Omega \times\right] 0, T$. Then we obtain a problem without a set of constraint equivalent to problem $\left(P_{\mathscr{F}}\right)$.

2. Preliminaries. Let $X$ be a real reflexive Banach space and let $X^{*}$ stand for its dual space with respect to the continuous pairing $\langle\cdots\rangle$. We may assume, without loss of generality, that $X$ and $X^{*}$ are locally uniformly convex, by virtue of the renorming theorem of Trojanski (cf. [5]). In particular, this implies that the duality mapping $J$ of $X$ into $X^{*}$ given by the following relations:

$$
\|J(u)\|_{X^{*}}=\|u\|_{X}, \quad\langle J(u), u\rangle=\|u\|_{X}^{2}, \quad \forall u \in X,
$$

is bijective bicontinuous. The norm convergence in $X$ and $X^{*}$ is denoted by $\rightarrow$, and the weak convergence by - . We consider a multi-values mapping (operator) $T$ from $X$ to $2^{X^{*}}$ (i.e., with values subsets of $X^{*}$ ). With each such map, we associate its graph

$$
G(T)=\left\{(u, w) \in X \times X^{*}: w \in T(u)\right\} .
$$

The multi-values mapping $T$ is said to be monotone $(T \in(\mathrm{M}))$ if for any pair of elements $\left(u_{1}, w_{1}\right),\left(u_{2}, w_{2}\right)$ in $G(T)$, we have the inequality

$$
\left\langle w_{1}-w_{2}, u_{1}-u_{2}\right\rangle \geq 0
$$

$T$ is said to be maximal monotone $(T \in(\mathrm{MM}))$ if it is monotone and maximal in the sense of graph inclusion among monotone multi-values mappings from $X$ to $2^{X^{*}}$. An equivalent version of the last clause is that for any $\left(u_{0}, w_{0}\right) \in X \times X^{*}$ for which

$$
\left\langle w_{0}-w, u_{0}-u\right\rangle \geq 0
$$

for all $(u, w) \in G(T)$, we have $\left(u_{0}, w_{0}\right) \in G(T)$. By Rockafellar theorem (see [9]) an equivalent statement is that the range of $T+\lambda J$ is all $X^{*}$ for all $\lambda>0$.

Let $T$ be a maximal monotone operator from $X$ to $2^{X^{*}}$.

(i) A sequence $\left\{T_{n}, n \in \mathbb{N}\right\}$ of maximal monotone operators from $X$ to $2^{X^{*}}$ is said to be graph-convergent to $T$ if for any $(u, w) \in G(T)$ there exists a sequence $\left(u_{n}, w_{n}\right) \in G\left(T_{n}\right)$ such that $u_{n} \rightarrow u$ in $X$ and $w_{n} \rightarrow w$ in $X^{*}$.

(ii) A family $\left\{T_{t}, t \in[0,1]\right\}$ of maximal monotone operators is said to be graphcontinuous if for any sequence $t_{n} \rightarrow t \in[0,1]$ and pair of elements $(u, w) \in G(T)$, 
there exists a sequence $\left(w_{n}, u_{n}\right) \in G\left(T_{t_{n}}\right)$ such that $u_{n} \rightarrow u$ in $X$ and $w_{n} \rightarrow w$ in $X^{*}$.

(iii) A family $\left\{T_{t}, t \in[0,1]\right\}$ of maximal monotone operators is said to be pointwise graph-continuous if for any sequence $t_{n} \rightarrow t \in[0,1]$ and any $(u, w) \in G(T)$, there exists a sequence $w_{n} \in T_{t_{n}}(u)$ such that $w_{n} \rightarrow w$ in $X^{*}$.

(iv) A family $\left\{T_{t}, t \in[0,1]\right\}$ of operators from $X$ to $2^{X^{*}}$ is said to be bounded if the range of a bounded subset of $X$ by $T_{t}$ is contained in a bounded subset of $X^{*}$ for all $t \in[0,1]$.

Let $T \in(\mathrm{MM})$ from $X \rightarrow 2^{X^{*}}$. Then for every $\lambda>0$ and every $u \in X$, there exists a unique element $R_{\lambda}^{T}(u)$ belonging to $D(T)$, domain of $T$, which is called the resolvant of index $\lambda$ of $T$, such that

$$
0 \in J\left(R_{\lambda}^{T}(u)-u\right)+\lambda T\left(R_{\lambda}^{T}(u)\right)
$$

We denote

$$
T_{\lambda}(u)=\frac{1}{\lambda} J\left(u-R_{\lambda}^{T}(u)\right), \quad \text { that is, } T_{\lambda}(u) \in T\left(R_{\lambda}^{T}(u)\right) .
$$

The operator $T_{\lambda}$ is called the Yosida approximation, it is a monotone continuous map from $X$ to $X^{*}$, graph-convergent to $T$ as $\lambda$ goes to zero and verify

$$
\left\|T_{\lambda}(u)\right\|_{X^{*}} \leq\|w\|_{X^{*}}, \quad \forall u \in X, w \in T(u) .
$$

The reader is referred to [2] for more details.

Now let $T$ be a linear densely defined monotone map from $D(T) \subset X$ to $X^{*}$, then a necessary and sufficient condition for $T \in(\mathrm{MM})$ is that $G(T)$ is a subspace of $X \times X^{*}$ and the conjugate $T^{*}$ of $T$ is monotone (cf. [7]).

We also need the following classes of mappings of monotone type. A mapping $T$ : $D(T) \subset X \rightarrow X^{*}$ is called

(v) quasimonotone $(T \in(\mathrm{QM}))$ if for any sequence $\left\{u_{n}\right\}$ in $D(L)$ with $u_{n} \rightarrow u$, we have $\lim \sup \left\langle T\left(u_{n}\right), u_{n}-u\right\rangle \geq 0$,

(vi) pseudomonotone $(T \in(\mathrm{PM}))$ if for any sequence $\left\{u_{n}\right\}$ in $D(L)$ with $u_{n} \rightarrow u$ and $\limsup \left\langle T\left(u_{n}\right), u_{n}-u\right\rangle \leq 0$, we have $\lim \left\langle T\left(u_{n}\right), u_{n}-u\right\rangle=0$, and if $u \in$ $D(T)$, then $T\left(u_{n}\right) \rightarrow T(u)$,

(vii) of class $\left(S_{+}\right)\left(T \in\left(S_{+}\right)\right)$if for any sequence $\left\{u_{n}\right\}$ in $D(L)$ with $u_{n} \rightarrow u$ and $\lim \sup \left\langle T\left(u_{n}\right), u_{n}-u\right\rangle \leq 0$, we have $u_{n} \rightarrow u$.

An example of mapping of class $\left(S_{+}\right)$is the duality map $J$, moreover, it is strictly monotone. Since $J^{-1}$ can be identified with the duality map from $X^{*}$ to $X^{* *}$, it is also of class $\left(S_{+}\right)$.

If we assume that all mappings are demicontinuous and defined in the whole space $X$, then $\left(S_{+}\right) \subset(\mathrm{PM}) \subset(\mathrm{QM})$ and $(M O N) \subset(\mathrm{PM})$. It is also important to observe that $\left(S_{+}\right)+(\mathrm{QM})$ is contained in $\left(S_{+}\right)$.

3. Construction of a degree function. Let $X$ be a real reflexive Banach space. We assume that $X$ and its conjugate $X^{*}$ are locally uniformly convex. Let $A$ be a bounded maximal monotone operator from $X$ to $2^{X^{*}}$ with $(0,0) \in G(A)$ and let $L$ be a linear maximal monotone map from $D(L) \subset X$ to $X^{*}$ such that $D(L)$ is dense in $X$. Since the 
graph of $L$ is a closed set in $X \times X^{*}, Y=D(L)$ equipped with the graph norm

$$
\|u\|_{Y}=\|u\|_{X}+\|L u\|_{X^{*}}, \quad u \in Y,
$$

becomes a real reflexive Banach space. We assume that $Y$ and its dual space $Y^{*}$ are also locally uniformly convex.

Let $j$ stand for the natural embedding of $Y$ to $X$ and let $j^{*}$ stand for its adjoint from $X^{*}$ to $Y^{*}$. For each open and bounded subset $G$ of $X$, let

$$
\begin{aligned}
\mathscr{F}_{G}= & \left\{L+A+S: \bar{G} \cap D(L) \rightarrow X^{*} \mid S\right. \text { is a bounded demicontinuous } \\
& \text { map of class } \left.\left(S_{+}\right) \text {with respect to } D(L) \text { from } \bar{G} \text { to } X^{*}\right\},
\end{aligned}
$$

and let

$$
\mathscr{H}_{G}=\left\{L+A_{t}+S(t): \bar{G} \cap D(L) \rightarrow X^{*} \mid A_{t}(0 \leq t \leq 1)\right. \text { is a bounded pointwise }
$$

graph-continuous homotopy of (MM) operators from $X$ to $2^{X^{*}}$ and $S(t)$ is a bounded homotopy of class $\left(S_{+}\right)$with respect to $D(L)$ from $\bar{G}$ to $X^{*}$ \}.

The family $S(t)$, with $0 \leq t \leq 1$, is called a homotopy of class $\left(S_{+}\right)$with respect to $D(L)$, if the conditions $u_{n} \rightarrow u, L u_{n} \rightarrow L u, t_{n} \rightarrow t$, and $\limsup \left\langle S\left(t_{n}\right) u_{n}, u_{n}-u\right\rangle \leq 0$ imply $u_{n} \rightarrow u$ and $S\left(t_{n}\right)\left(u_{n}\right) \rightarrow S(t)(u)$. Note that the class $\mathscr{H}_{G}$ includes all affine homotopies $L+(1-t)\left(A_{1}+S_{1}\right)+t\left(A_{2}+S_{2}\right)$ with $\left(L+A_{i}+S_{i}\right) \in \mathscr{F}_{G}, i=1$, 2. In order to find suitable approximations for mappings $F \in \mathscr{F}_{G}$, let

$$
\hat{L}=j^{*} \circ L \circ j,
$$

which is obviously a bounded linear monotone map from $Y$ to $Y^{*}$. Similarly, let

$$
\hat{S}(t)=j^{*} \circ S(t) \circ j: j^{-1}(\bar{G}) \longrightarrow Y^{*}
$$

whenever $S(t)$ is a homotopy from $\bar{G}$ to $X^{*}$, and for every $\lambda>0$,

$$
\hat{A}_{t, \lambda}=j^{*} \circ A_{t, \lambda} \circ j,
$$

where $A_{t, \lambda}$ is the Yosida approximation of index $\lambda$ of the operator $A_{t}$. Since $j$ is continuous from $Y$ to $X, j^{-1}(\bar{G})=\bar{G} \cap D(L)$ is closed and $j^{-1}(G)=G \cap D(L)$ is open in $Y$. It is easy to check that

$$
\overline{j^{-1}(G)} \subset j^{-1}(\bar{G}) ; \quad \partial\left(j^{-1}(G)\right) \subset j^{-1}(\partial G) .
$$

Note that we have the same notation for closures and boundaries in both $X$ and $Y$. In what follows, we also need the map $\mathrm{M}: Y \rightarrow Y^{*}$ defined by

$$
(\mathrm{M}(u), v)=\left\langle L v, J^{-1}(L u)\right\rangle, \quad u, v \in Y,
$$

where $(\cdot, \cdot)$ denotes the pairing between $Y$ and $Y^{*}$ and $J^{-1}$ is the inverse of the duality map $J: X \rightarrow X^{*}$. In fact, for all $u \in Y$ for which $\mathrm{M}(u) \in j^{*}\left(X^{*}\right)$, we have $J^{-1}(L u) \in$ $D\left(L^{*}\right)$ and by (3.6),

$$
\mathrm{M}(u)=j^{*} L^{*} J^{-1}(L u) .
$$

We need this representation later in proving Lemma 3.4. For each admissible map $F \in \mathscr{F}_{G}$ or homotopy $F(t) \in \mathscr{H}_{G}$ and for each $\lambda>0$, we define

$$
\hat{F}_{\lambda}=\hat{L}+\hat{A}_{\lambda}+\hat{S}+\lambda \mathrm{M}, \quad \hat{F}_{\lambda}(t)=\hat{L}+\hat{A}_{t, \lambda}+\hat{S}(t)+\lambda \mathrm{M} .
$$


LEMMA 3.1. Let $\left\{T_{n}, n \in \mathbb{N}\right\}$ and $T$ be a sequence of maximal monotone operators from $X$ to $2^{X^{*}}$. Then the following properties hold.

(a) If $T_{n}$ is graph-convergent to $T$, then for any sequence $\left\{u_{n}\right\} \subset X$ such that $u_{n} \rightarrow u$ in $X$, we have $T_{n, \lambda}\left(u_{n}\right) \rightarrow T_{\lambda}(u)$ in $X^{*}$ for every $\lambda>0$. Moreover, if the sequence $\left\{T_{n}\right\}$ is bounded, we have $T_{n, \lambda_{n}}\left(u_{n}\right) \rightarrow \beta \in T(u)$ for a subsequence $\left\{u_{n}\right\}$ and any sequence $\left\{\lambda_{n} ; \lambda_{n} \geq 0\right\}$ with $\lambda_{n} \rightarrow 0$.

(b) If the sequence $\left\{T_{n}\right\}$ is a pointwise graph-continuous to $T$, then we have $T_{n, \lambda_{n}}(u) \rightarrow T^{\circ}(u)$ for any sequence $\left\{\lambda_{n} ; \lambda_{n} \geq 0\right\}$ with $\lambda_{n} \rightarrow 0$, where $T^{\circ}(u)$ is the element of minimal norm of the closed convex subset $T(u)$ of $X^{*}$.

Proposition 3.2 (see [5]). Let $\left\{T_{n}\right\}, T$ be a sequence of maximal monotone operators with $T_{n}$ is graph-convergent to $T$ and let $\left\{\left(\alpha_{n}, \beta_{n}\right)\right\}$ be a sequence in $G\left(T_{n}\right)$ such that $\alpha_{n} \longrightarrow \alpha$ in $X, \beta_{n} \longrightarrow \beta$ in $X^{*}$ and $\lim \sup \left\langle\beta_{n}, \alpha_{n}\right\rangle \leq\langle\beta, \alpha\rangle$. Then we have $\beta \in T(\alpha)$ and $\left\langle\beta_{n}, \alpha_{n}\right\rangle \rightarrow\langle\beta, \alpha\rangle$.

Proof OF Lemma 3.1. In this proof, we use the same notation of norm $\|\cdot\|$ of $X$ and $X^{*}$.

(a) We first notice that the sequences $\left\{R_{\lambda}^{T_{n}}\left(u_{n}\right)\right\}$ and $\left\{T_{n, \lambda}\left(u_{n}\right)\right\}$ are bounded. Let $\left(u_{0}, w_{0}\right) \in T$, then there exists $\left(u_{0 n}, w_{0 n}\right) \in G\left(T_{n}\right)$ such that $\left(u_{0 n}, w_{0 n}\right) \rightarrow\left(u_{0}, w_{0}\right)$. From monotonicity of $T_{n}$ and relations (2.6),

$$
\left\langle\frac{1}{\lambda} J\left(u_{n}-R_{\lambda}^{T_{n}}\left(u_{n}\right)\right)-w_{0 n}, R_{\lambda}^{T_{n}}\left(u_{n}\right)-u_{0 n}\right\rangle \geq 0 .
$$

Thus

$$
\left\langle\frac{1}{\lambda} J\left(u_{n}-R_{\lambda}^{T_{n}}\left(u_{n}\right)\right)-w_{0 n}, R_{\lambda}^{T_{n}}\left(u_{n}\right)-u_{n}+u_{n}-u_{0 n}\right\rangle \geq 0
$$

that is,

$$
\begin{aligned}
\left\|u_{n}-R_{\lambda}^{T_{n}}\left(u_{n}\right)\right\|^{2} \leq & \left\|u_{n}-R_{\lambda}^{T_{n}}\left(u_{n}\right)\right\|\left(\left\|u_{n}-u_{0 n}\right\|+\lambda\left\|w_{0 n}\right\|\right)+\lambda\left\|w_{0 n}\right\|\left\|u_{n}-u_{0 n}\right\| \\
& \cdot\left\|u_{n}-R_{\lambda}^{T_{n}}\left(u_{n}\right)\right\| \leq 2\left(\left\|u_{n}-u_{0 n}\right\|+\lambda\left\|w_{0 n}\right\|\right) .
\end{aligned}
$$

Hence, the sequences $\left\{R_{\lambda}^{T_{n}}\left(u_{n}\right)\right\}$ and $\left\{T_{n, \lambda}\left(u_{n}\right)\right\}$ being bounded in reflexive Banach spaces $X$ and $X^{*}$, respectively. We can extract a subsequence (still denoted by $\left\{u_{n}\right\}$ ) such that $R_{\lambda}^{T_{n}}\left(u_{n}\right) \rightarrow \alpha$ in $X$ and $T_{n, \lambda}\left(u_{n}\right) \rightarrow \beta$ in $X^{*}$.

Let us identify $\alpha$ and $\beta$. By monotonicity of $J$, for every integers $n, m$,

$$
\left\langle\frac{1}{\lambda} J\left(u_{n}-R_{\lambda}^{T_{n}}\left(u_{n}\right)\right)-\frac{1}{\lambda} J\left(u_{m}-R_{\lambda}^{T_{m}}\left(u_{m}\right)\right),\left(u_{n}-R_{\lambda}^{T_{n}}\left(u_{n}\right)\right)-\left(u_{m}-R_{\lambda}^{T_{m}}\left(u_{m}\right)\right)\right\rangle \geq 0
$$

Thus

$$
\begin{aligned}
& \left\langle T_{n, \lambda}\left(u_{n}\right), R_{\lambda}^{T_{n}}\left(u_{n}\right)\right\rangle+\left\langle T_{m, \lambda}\left(u_{m}\right), R_{\lambda}^{T_{m}}\left(u_{m}\right)\right\rangle \\
& \quad \leq\left\langle T_{n, \lambda}\left(u_{n}\right)-T_{m, \lambda}\left(u_{m}\right), u_{n}-u_{m}\right\rangle+\left\langle T_{n, \lambda}\left(u_{n}\right), R_{\lambda}^{T_{m}}\left(u_{m}\right)\right\rangle+\left\langle T_{m, \lambda}\left(u_{m}\right), R_{\lambda}^{T_{n}}\left(u_{n}\right)\right\rangle .
\end{aligned}
$$

Let us first fix $m$ and let $n$ go to $+\infty$, next let $m$ go to $+\infty$, then we have

$$
\limsup \left\langle T_{n, \lambda}\left(u_{n}\right), R_{\lambda}^{T_{n}}\left(u_{n}\right)\right\rangle \leq\langle\beta, \alpha\rangle .
$$


Applying Proposition 3.2 to (3.14) with $\alpha_{n}=R_{\lambda}^{T_{n}}\left(u_{n}\right), \beta_{n}=T_{n, \lambda}\left(u_{n}\right)$, we obtain

$$
\beta \in T(\alpha), \quad\left\langle T_{n, \lambda\left(u_{n}\right)}, R_{\lambda}^{T_{n}}\left(u_{n}\right)\right\rangle \longrightarrow\langle\beta, \alpha\rangle .
$$

By applying Proposition 3.2 to the operator $J$ with $\alpha_{n}=(1 / \lambda)\left(u_{n}-R_{\lambda}^{T_{n}}\left(u_{n}\right)\right)$ and $\beta_{n}=$ $(1 / \lambda) J\left(u_{n}-R_{\lambda}^{T_{n}}\left(u_{n}\right)\right)$, and taking into account that $\left\langle\beta_{n}, \alpha_{n}\right\rangle \rightarrow\langle\beta,(1 / \lambda)(u-\alpha)\rangle$, we obtain

$$
\beta=\frac{1}{\lambda} J(u-\alpha), \quad\left\|u_{n}-R_{\lambda}^{T_{n}}\left(u_{n}\right)\right\| \longrightarrow\|u-\alpha\| .
$$

Hence $R_{\lambda}^{T_{n}}\left(u_{n}\right) \rightarrow \alpha$. Combining (3.15) and (3.16), we have

$$
0 \in T(\alpha)+\frac{1}{\lambda} J(\alpha-u)
$$

and from the unicity of the solution of (3.17), we conclude that

$$
\alpha=R_{\lambda}^{T}(u) .
$$

Hence $R_{\lambda}^{T_{n}}\left(u_{n}\right) \rightarrow R_{\lambda}^{T}(u)$. Consequently, $T_{n, \lambda}\left(u_{n}\right) \rightarrow T_{\lambda}(u)$.

Now let $\lambda_{n} \rightarrow 0$. For $(u, w) \in G(T)$ there exists a sequence $\left(u_{0 n}, w_{n}\right) \in G\left(T_{n}\right)$ such that $u_{0 n} \rightarrow u_{0}$ in $X$ and $w_{n} \rightarrow w_{0}$ in $X^{*}$. The same estimation as (3.11) yields

$$
\left\|u_{n}-R_{\lambda_{n}}^{T_{n}}\left(u_{n}\right)\right\| \leq 2\left(\left\|u_{n}-u_{0 n}\right\|+\lambda_{n}\left\|w_{n}\right\|\right)
$$

implies that $R_{\lambda_{n}}^{T_{n}}\left(u_{n}\right) \rightarrow u$ as $n$ goes to infinity. Since $\left\{T_{n, \lambda_{n}}\left(u_{n}\right)\right\}$ is bounded in reflexive Banach space $X^{*}$, we can extract a subsequence (still denoted by $\left\{u_{n}\right\}$ ) such that $T_{n, \lambda_{n}}\left(u_{n}\right) \rightarrow \beta$. Applying Proposition 3.2 to the sequence of operators $\left\{T_{n}\right\}$ with $\alpha_{n}=R_{\lambda_{n}}^{T_{n}}\left(u_{n}\right)$ and $\beta_{n}=T_{n, \lambda_{n}}\left(u_{n}\right)$, we obtain $\beta \in T(u)$.

(b) Let $\lambda_{n} \rightarrow 0$ and $w \in T(u)$, then the property pointwise graph-continuous of $T_{n}$ implies that there exists a sequence $w_{n} \in T_{n}(u)$ such that $w_{n} \rightarrow w$ in $X^{*}$. By monotonicity of $T_{n}$,

$$
\left\langle w_{n}-\frac{1}{\lambda_{n}} J\left(u-R_{\lambda_{n}}^{T_{n}}(u)\right), u-R_{\lambda_{n}}^{T_{n}}(u)\right\rangle \geq 0 .
$$

Thus

$$
\frac{1}{\lambda_{n}}\left\|u-R_{\lambda_{n}}^{T_{n}}(u)\right\| \leq\left\|w_{n}\right\|
$$

The relation above implies that $R_{\lambda_{n}}^{T_{n}}(u) \rightarrow u$ and

$$
\limsup \left\|T_{n, \lambda_{n}}(u)\right\| \leq\|w\|, \quad \forall w \in T(u) .
$$

Hence,

$$
\limsup \left\|T_{n, \lambda_{n}}(u)\right\| \leq\left\|T^{\circ}(u)\right\| .
$$

Then there exists a subsequence (still denoted by $\left\{u_{n}\right\}$ ) such that

$$
T_{n, \lambda_{n}}(u) \longrightarrow \beta \text { in } X^{*} .
$$


Applying Proposition 3.2 to the sequence of operators $\left\{T_{n}\right\}$ with $\alpha_{n}=R_{\lambda_{n}}^{T_{n}}(u)$ and $\beta_{n}=T_{n, \lambda_{n}}(u)$, we obtain $\beta \in T(u)$, which implies that $\|\beta\| \geq\left\|T^{\circ}(u)\right\|$. Since $T_{n, \lambda_{n}}(u) \rightarrow \beta$, then

$$
\liminf \left\|T_{n, \lambda_{n}}(u)\right\| \geq\|\beta\| .
$$

Combining (3.23) and (3.25), we have

$$
\left\|T_{n, \lambda_{n}}\left(u_{n}\right)\right\| \longrightarrow\left\|T^{\circ}(u)\right\|=\|\beta\| .
$$

Now, by definition of $T^{\circ}(u)$ we conclude that $\beta=T^{\circ}(u)$. Finally, from (3.24) and (3.26) we have

$$
T_{n, \lambda_{n}}(u) \longrightarrow T^{\circ}(u)
$$

LEMMA 3.3. If $F(t) \in \mathscr{H}_{G}$ and $\lambda>0$, then $\hat{F}_{\lambda}(t)$ is a bounded homotopy of class $\left(S_{+}\right)$ from $j^{-1}(\bar{G}) \subset Y$ to $Y^{*}$. In particular, for each $\lambda>0, \hat{F}_{\lambda}$ is a bounded demicontinuous map of class $\left(S_{+}\right)$from $j^{-1}(\bar{G}) \subset Y$ to $Y^{*}$.

Proof. Assume $F(t) \in \mathscr{H}_{G}$ and $\lambda>0$. Let $\left\{u_{n}\right\} \subset \bar{G} \cap D(L)$ with $u_{n} \rightarrow u$ in $Y, t_{n} \rightarrow t$, and $\limsup \left(\hat{F}_{\lambda}\left(t_{n}\right)\left(u_{n}\right), u_{n}-u\right) \leq 0$. Then $u_{n} \rightarrow u$ in $X, L u_{n} \rightarrow L u$ in $X^{*}$ and since $A_{t_{n}, \lambda}(u) \rightarrow A_{t, \lambda}(u)$,

$$
\begin{aligned}
\limsup \{ & \left\langle L u_{n}-L u, u_{n}-u\right\rangle+\left\langle A_{t_{n}, \lambda}\left(u_{n}\right)-A_{t_{n}, \lambda}(u), u_{n}-u\right\rangle \\
& \left.+\left\langle S\left(t_{n}\right)\left(u_{n}\right), u_{n}-u\right\rangle+\lambda\left\langle L u_{n}-L u, J^{-1}\left(L u_{n}\right)-J^{-1}(L u)\right\rangle\right\} \leq 0 .
\end{aligned}
$$

Since $L, J^{-1}$, and $A_{t_{n}, \lambda}$ are monotone we conclude that

$$
\limsup \left\langle S\left(t_{n}\right)\left(u_{n}\right), u_{n}-u\right\rangle \leq 0 .
$$

By the $\left(S_{+}\right)$-property of $S(t)$ we obtain $u_{n} \rightarrow u$ in $X$ and $S\left(t_{n}\right)\left(u_{n}\right) \rightarrow S(t)(u)$ in $X^{*}$. Then by Lemma 3.1(a),

$$
A_{t_{n}, \lambda}\left(u_{n}\right) \longrightarrow A_{t, \lambda}(u)
$$

Therefore,

$$
\lim \left\langle L u_{n}-L u, J^{-1}\left(L u_{n}\right)-J^{-1}(L u)\right\rangle=0
$$

implying, by the $\left(S_{+}\right)$-property of $J^{-1}$, that $L u_{n} \rightarrow L u$ in $X^{*}$, then the assertion follows.

Let $F(t) \in \mathscr{H}_{G}$ and let $\{h(t), 0 \leq t \leq 1\}$ be a continuous curve in $X^{*}$. We denote

$$
\begin{gathered}
K=\left\{u \in j^{-1}(\bar{G}) \mid s \hat{F}_{\lambda}(t)(u)+(1-s) \hat{F}_{\delta}(t)(u)=j^{*} h(t)\right. \\
\quad \text { for some } \lambda, \delta>0, \text { and } s, t \in[0,1]\} .
\end{gathered}
$$

Note that $j(K) \subset \bar{G}$ implying that $K$ is bounded in $X$. The fact that $K$ is bounded also in $Y$ follows from the following.

LEMMA 3.4. There exists a constant $R>0$, independent of $\lambda, \delta, s$, and $t$, such that $K \subset B_{R}(Y)=\left\{v \in Y \mid\|v\|_{Y}<R\right\}$. 
Proof. Without loss of generality, we may assume that $h(t) \equiv 0$. Let $u \in K$ be arbitrary. Then for some $\lambda, \delta>0$ and $s, t \in[0,1]$,

$$
\langle L u, v\rangle+\langle w, v\rangle+\langle S(t)(u), v\rangle+(s \lambda+(1-s) \delta)\left\langle L^{*} J^{-1}(L u), v\right\rangle=0, \quad \forall v \in D(L),
$$

where $w=s A_{t, \lambda}(u)+(1-s) A_{t, \delta}(u)$. Observe that $J^{-1}(L u) \in D\left(L^{*}\right)$ since $\mathrm{M}(u) \in$ $j^{*}\left(X^{*}\right)$. Since $D(L)$ is dense in $X$, equation (3.33) holds for all $v \in X$. Hence we can insert $v=J^{-1}(L u)$ to get

$$
\begin{aligned}
\left\langle L u, J^{-1}(L u)\right\rangle & +\left\langle w, J^{-1}(L u)\right\rangle+\left\langle S(t)(u), J^{-1}(L u)\right\rangle \\
& +(s \lambda+(1-s) \delta)\left\langle L^{*} J^{-1}(L u), J^{-1}(L u)\right\rangle=0 .
\end{aligned}
$$

Recalling that $L^{*}$ is monotone, we obtain

$$
\|L u\|_{X^{*}}^{2} \leq\left\|s A_{t, \lambda}(u)+(1-s) A_{t, \delta}(u)+S(t)(u)\right\|_{X^{*}}\left\|J^{-1}(L u)\right\|_{X^{*}}
$$

Since $\left\|J^{-1}(L u)\right\|_{X}=\|L u\|_{X^{*}}$ and since $A_{t}$ and $S(t)$ are bounded homotopies from a bounded set $\bar{G} \subset X$ we conclude that

$$
\|L u\|_{X^{*}} \leq c
$$

for some positive constant $c$ independent of $\lambda, \delta, s$ and $t$, this completes the proof.

The relationship between $F(t) \in \mathscr{H}_{G}$ and its approximation $\hat{F}_{\lambda}(t)$ is shown by the following.

LEMMA 3.5. Let $\Omega \subset \bar{G}$ be a closed set, $F(t) \in \mathscr{H}_{G}$ an admissible homotopy, and $h(t)$ a continuous curve in $X^{*}$ such that

$$
h(t) \notin F(t)(\Omega \cap D(L)), \quad \forall t \in[0,1] .
$$

Then there exists $\lambda_{0}>0$ such that

$$
j^{*} h(t) \notin \hat{F}_{\lambda}(t)\left(j^{-1}(\Omega)\right), \quad \forall t \in[0,1], 0<\lambda<\lambda_{0} .
$$

Proof. We may assume that $h(t) \equiv 0$. We argue by a contradiction. Let us assume that there exist the sequences $\left\{\lambda_{n}\right\},\left\{t_{n}\right\}$, and $\left\{u_{n}\right\} \subset j^{-1}(\Omega)$ such that $\lambda_{n} \rightarrow 0^{+}$, $t_{n} \rightarrow t \in[0,1]$, and

$$
\hat{L} u_{n}+\hat{A}_{t_{n}, \lambda_{n}}\left(u_{n}\right)+\hat{S}\left(t_{n}\right)\left(u_{n}\right)+\lambda_{n} \mathrm{M}\left(u_{n}\right)=0 .
$$

By Lemma 3.4 the sequence $\left\{u_{n}\right\}$ is bounded in $Y$ implying that $u_{n} \rightarrow u$ in $X$ and $L u_{n} \longrightarrow L u$ in $X^{*}$ with $u \in D(L)$, for a subsequence. Using the fact that $L, A_{t_{n}, \lambda_{n}}$, $J^{-1}$ are monotone and $A_{t_{n}, \lambda_{n}}(u) \rightarrow A_{t}^{\circ}(u)$ we get from (3.39),

$$
\begin{aligned}
\limsup \left\langle S\left(t_{n}\right)\left(u_{n}\right), u_{n}-u\right\rangle=\limsup \{ & -\left\langle L u_{n}-L u, u_{n}-u\right\rangle \\
& -\left\langle A_{t_{n}, \lambda_{n}}\left(u_{n}\right)-A_{t_{n}, \lambda_{n}}(u), u_{n}-u\right\rangle \\
& \left.-\lambda_{n}\left\langle L u_{n}-L u, J^{-1}\left(L u_{n}\right)-J^{-1}(L u)\right\rangle\right\} \leq 0 .
\end{aligned}
$$


Since $S(t)$ is a homotopy of class $\left(S_{+}\right)$, then $u_{n} \rightarrow u$ in $X$ and $S\left(t_{n}\right)\left(u_{n}\right) \rightarrow S(t)(u)$ in $X^{*}$ with $u \in \Omega$. By (3.39),

$$
\left(\hat{L} u_{n}, v\right)+\left(\hat{A}_{t_{n}, \lambda_{n}}\left(u_{n}\right), v\right)+\left(\hat{S}\left(t_{n}\right)\left(u_{n}\right), v\right)+\lambda_{n}\left(\mathrm{M}\left(u_{n}\right), v\right)=0
$$

for all $v \in Y$ and $n \in \mathbb{N}$. Since $A_{t_{n}, \lambda_{n}}\left(u_{n}\right) \rightarrow w_{t} \in A_{t}(u)$ for a subsequence $\left\{u_{n}\right\}$ (Lemma 3.1), then as $n$ tends to $+\infty$,

$$
\langle L u, v\rangle+\left\langle w_{t}, v\right\rangle+\langle S(t)(u), v\rangle=0, \quad \forall v \in D(L) .
$$

Now $D(L)$ is dense in $X$,

$$
0 \in L u+A_{t}(u)+S(t)(u)
$$

with $u \in \Omega \cap D(L)$, which contradicts our assumption. Hence the proof is complete.

By choosing $\Omega=\partial G, F(t)=F \in \mathscr{F}_{G}$, and $h(t)=h \in X^{*}$ as in Lemma 3.5, the condition $h \notin(L+A+S)(\partial G \cap D(L))$ implies that there exists $\lambda_{0}>0$ such that

$$
j^{*} h \notin\left(\hat{L}+\hat{A_{\lambda}}+\hat{S}+\lambda \mathrm{M}\right)\left(j^{-1}(\partial G)\right), \quad \forall 0<\lambda<\lambda_{0} .
$$

Recalling (3.5), we also have

$$
j^{*} h \notin \hat{F}_{\lambda}\left(\partial\left(j^{-1}(G)\right)\right), \quad \forall 0<\lambda<\lambda_{0} .
$$

Moreover, by Lemma 3.4 there exists a constant $R>0$, independent of $\lambda$, such that

$$
j^{*} h \notin \hat{F}_{\lambda}(u), \quad \forall u \in \bar{G},\|u\| \geq R, \lambda>0 .
$$

Denoting $G_{R}(Y)=j^{-1}(G) \cap B_{R}(Y)$, we therefore have

$$
j^{*} h \notin \hat{F}_{\lambda}(\partial G(Y))
$$

for all $\lambda$ with $0<\lambda<\lambda_{0}$. Since $\hat{F}_{\lambda}=\hat{L}+A_{\lambda}+\hat{S}+\lambda$ M is a map of class $S_{+}$from $j^{-1}(\bar{G}) \subset Y$ to $Y^{*}$ by Lemma 3.3, the value of the unique topological degree (see [5]),

$$
d_{S_{+}}\left(\hat{F}_{\lambda}, G_{R}(Y), j^{*} h\right)
$$

is well defined for all $0<\lambda<\lambda_{0}$.

LemmA 3.6. Let $h \in X$ such that $h \notin(L+A+S)(\partial G \cap D(L))$, then there exists a constant $\lambda^{\prime}>0$ such that

$$
d_{S_{+}}\left(\hat{L}+\hat{A}_{\lambda}+\hat{S}+\lambda \mathrm{M}, G_{R}(Y), j^{*} h\right)=\text { constant }
$$

for all $\lambda$ with $0<\lambda<\lambda^{\prime}$.

Proposition 3.7 (see [5]). Let $X$ be a reflexive Banach space, $\left\{A_{t}, t \in[0,1]\right\} a$ graph-continuous family of maximal monotone operators from $X$ to $2^{X^{*}}$ with $(0,0) \in$ $G(A)$ for all $t$. Let $\left\{u_{n}\right\}$ be a sequence in $X, u_{n} \rightarrow u$ and for sequences $\left\{t_{n}\right\} \in[0,1]$, $t_{n} \rightarrow t, 0<\lambda_{n}, 0<\delta_{n}, \lambda_{n} \rightarrow 0$, and $\delta_{n} \rightarrow 0$, let

$$
y_{n}=A_{t_{n}, \lambda_{n}}\left(u_{n}\right), \quad z_{n}=A_{t_{n}, \delta_{n}}\left(u_{n}\right) .
$$


Suppose further that for another sequence $\left\{s_{n}\right\}$ in $[0,1]$, and for

$$
w_{n}=\left(1-s_{n}\right) y_{n}+s_{n} z_{n},
$$

we have $w_{n} \rightarrow w$ in $X^{*}$. If $\limsup \left\langle w_{n}, u_{n}\right\rangle \leq\langle w, u\rangle$, then $w \in A_{t}(u)$ and $\left\langle w_{n}, u_{n}\right\rangle \rightarrow\langle w, u\rangle$.

Proof OF Lemma 3.6. In order to employ the homotopy argument of the degree $d_{S_{+}}$, we show the existence of $\lambda^{\prime}$ such that

$$
j^{*} h \notin\left[(1-s) \hat{F}_{\lambda}+s \hat{F}_{\delta}\right]\left(j^{-1}(\partial G)\right)
$$

for all $s \in[0,1], 0<\lambda \leq \lambda^{\prime}, 0<\delta \leq \lambda^{\prime}$. Indeed, we assume the contrary, that is, there exist sequences $\left\{u_{n}\right\} \subset j^{-1}(\partial G),\left\{s_{n}, s\right\}$ in $[0,1]$ and two sequences of positive numbers $\lambda_{n}, \delta_{n} \rightarrow 0$ such that

$$
j^{*} h=\hat{L} u_{n}+\left(1-s_{n}\right) \hat{A}_{\lambda_{n}}\left(u_{n}\right)+s_{n} \hat{A}_{\delta_{n}}\left(u_{n}\right)+\hat{S}\left(u_{n}\right)+\left(\left(1-s_{n}\right) \lambda_{n}+s_{n} \delta_{n}\right) \mathrm{M}\left(u_{n}\right) .
$$

Since $A$ is a bounded operator from $X$ to $2^{X^{*}}$, then the sequence

$$
w_{n}=\left(1-s_{n}\right) \hat{A}_{\lambda_{n}}\left(u_{n}\right)+s_{n} \hat{A}_{\delta_{n}}\left(u_{n}\right)
$$

is bounded in $X^{*}$. We may assume that $w_{n} \rightarrow w$ in $X^{*}$ and $u_{n} \rightarrow u$ in $X$, we have

$$
\begin{aligned}
\limsup \left\langle w_{n}, u_{n}-u\right\rangle & =\limsup \left\langle-S\left(u_{n}\right)-L u_{n}, u_{n}-u\right\rangle \\
& \leq \limsup \left\langle-S\left(u_{n}\right), u_{n}-u\right\rangle+\limsup \left\langle-L u_{n}, u_{n}-u\right\rangle \\
& \leq \limsup \left\langle-S\left(u_{n}\right), u_{n}-u\right\rangle \\
& =-\liminf \left\langle S\left(u_{n}\right), u_{n}-u\right\rangle \leq 0,
\end{aligned}
$$

that is,

$$
\limsup \left\langle w_{n}, u_{n}\right\rangle \leq\langle w, u\rangle .
$$

Proposition 3.7 implies that $\left\langle w_{n}, u_{n}\right\rangle \rightarrow\langle w, u\rangle$ and $w \in A(u)$. Hence

$$
\limsup \left\langle S\left(u_{n}\right), u_{n}-u\right\rangle \leq 0 \text {. }
$$

The property of class $\left(S_{+}\right)$of the operator $S$ implies that $u_{n} \rightarrow u \in j^{-1}(\partial G)$ and $S\left(u_{n}\right) \rightarrow S(u)$. Applying $v$ to the two sides of the equality (3.53) and tending $n$ to infinity, then we have

$$
\langle h, v\rangle=\langle L u, v\rangle+\langle w, v\rangle+\langle S(u), v\rangle, \quad \forall v \in D(L) .
$$

Since $D(L)$ is dense in $X$,

$$
h=L u+S(u)+w,
$$

where $w \in A(u)$ and $u \in j^{-1}(\partial G)$. Which contradicts the relation (3.52). That is,

$$
j^{*} h \notin\left((1-s) \hat{F}_{\lambda}+s \hat{F}_{\delta}\right) \partial\left(G_{R}(Y)\right)
$$


for all $\delta, \lambda, 0<\delta<\lambda^{\prime}, 0<\lambda<\lambda^{\prime}, s \in[0,1]$ and $R$ is a constant satisfying Lemma 3.4. By the invariance under homotopy property of $\left(S_{+}\right)$-degree, we obtain

$$
d_{S_{+}}\left(\hat{F}_{\lambda}, G_{R}(Y), j^{*} h\right)=d_{S_{+}}\left(\hat{F}_{\delta}, G_{R}(Y), j^{*} h\right)=\text { constant }
$$

for all $\lambda, 0<\lambda<\lambda^{\prime}$

Consequently, it is relevant to define a function $D$ by

$$
D(L+A+S, G, h)=\lim _{\lambda \rightarrow 0^{+}} d_{S_{+}}\left(\hat{L}+\hat{A}_{\lambda}+\hat{S}+\lambda \mathrm{M}, G_{R}(Y), j^{*} h\right)
$$

whenever $h \notin(L+A+S)(\partial G \cap D(L))$ and $R$ is sufficiently large (satisfying Lemma 3.4).

THEOREM 3.8. Let $X$ be a real reflexive Banach space, $L$ a linear maximal monotone densely defined map from $D(L) \subset X$ to $X^{*}, G$ an open bounded subset in $X$ and $\mathscr{F}_{G}$ the class of admissible operators. Then there exists a function topological degree $D$ defined from $\left\{(F, G, h): F \in \mathscr{F}_{G}, G\right.$ an open bounded subset in $X$ and $\left.h \notin F(\partial G \cap D(L))\right\}$ to $\mathbb{Z}$. Satisfying the following properties:

$\left(D_{1}\right)$ If $D(F, G, h) \neq 0$, then there exists $u \in G \cap D(L)$ such that $h \in F(u)$.

$\left(\mathrm{D}_{2}\right)$ (Additivity of domain.) If $G^{1}$ and $G^{2}$ are open disjoint subsets of $G$ and $h \notin$ $F\left[\left(\bar{G} \backslash\left(G^{1} \cup G^{2}\right)\right) \cap D(L)\right]$, then

$$
D(F, G, h)=D\left(F, G^{1}, h\right)+D\left(F, G^{2}, h\right) .
$$

$\left(\mathrm{D}_{3}\right)$ (Invariance under admissible homotopies.) If $F(t) \in \mathscr{H}_{G}$ and $h(t) \notin F(t)(\partial G \cap$ $D(L))$ for all $t \in[0,1]$, where $h(t)$ is a continuous curve in $X^{*}$, then

$$
D(F(t), G, h(t))=\text { constant }, \quad \forall t \in[0,1] .
$$

$\left(\mathrm{D}_{4}\right) L+J$ is the normalising map, that is,

$$
D(L+J, G, h)=1, \quad \text { whenever } h \in(L+J)(G \cap D(L)) .
$$

Proof. It is the same as the one given in [4] (properties of the degree).

4. Existence result. Let $X$ be a real reflexive Banach space, $L$ a linear maximal monotone map from $D(L) \subset X$ to $X^{*}$ with $D(L)$ dense in $X$, and $A$ a bounded maximal monotone operator from $X$ to $2^{X^{*}}$ with $(0,0) \in G(A)$ and $G$ an open bounded subset of $X$. Let

$$
\begin{aligned}
\mathscr{F}_{G}(\mathrm{PM})= & \{F=L+A+S: S \text { is a bounded demicontinuous } \\
& \text { pseudomonotone with respect to } \left.D(L) \text { map from } \bar{G} \text { to } X^{*}\right\}, \\
\mathscr{F}_{G}(\mathrm{QM})=\{ & \{F=L+A+S: S \text { is a bounded demicontinuous } \\
& \text { quasimonotone with respect to } \left.D(L) \text { map from } \bar{G} \text { to } X^{*}\right\} .
\end{aligned}
$$

For a given $h \in X^{*}$, we are interested in the solvability of the equation

$$
h \in L u+A(u)+S(u), \quad u \in D(L) .
$$


LEMmA 4.1. Let $G$ be a convex open bounded subset in $X$ and $F \in \mathscr{F}_{G}$. Then $F(\bar{G} \cap$ $D(L))$ is closed in $X^{*}$.

Proof. Let $\left\{z_{n}\right\}$ be a sequence in $(L+A+S)(\bar{G} \cap D(L))$ with $z_{n}=L u_{n}+w_{n}+$ $S\left(u_{n}\right) \rightarrow z, u_{n} \in \bar{G} \cap D(L)$ and $w_{n} \in A\left(u_{n}\right)$. Since subsequences $\left\{u_{n}\right\},\left\{S\left(u_{n}\right)\right\}$, and $\left\{w_{n}\right\}$ are bounded in reflexive Banach spaces $X$ and $X^{*}$, then there exists a subsequence (still denoted by $\left\{u_{n}\right\}$ ), such that $u_{n} \rightarrow u$ in $X$ with $u \in \bar{G}, w_{n} \rightarrow w$ in $X^{*}$ and $S\left(u_{n}\right) \longrightarrow s$ in $X^{*}$. Hence $L u_{n} \longrightarrow z-w-s$. Since also $G(L)$ is weakly closed, we have $u \in D(L)$ and $L u=z-w-s$. Consequently,

$$
\begin{aligned}
\limsup \left\langle S\left(u_{n}\right), u_{n}-u\right\rangle & =\limsup \left\langle z_{n}-L u_{n}-w_{n}, u_{n}-u\right\rangle \\
& =\limsup \left\langle-L u_{n}-w_{n}, u_{n}-u\right\rangle \\
& =-\liminf \left\{\left\langle L u_{n}-L u, u_{n}-u\right\rangle+\left\langle w_{n}-\bar{w}, u_{n}-u\right\rangle\right\} \leq 0,
\end{aligned}
$$

where $\bar{w}$ is an element of $A(u)$. Since $S$ is pseudomonotone, we obtain $S\left(u_{n}\right) \longrightarrow S(u)$ $=s$ and $\left\langle S\left(u_{n}\right), u_{n}\right\rangle \rightarrow\langle S(u), u\rangle$. Therefore, $\limsup \left\langle w_{n}, u_{n}\right\rangle \leq\langle w, u\rangle$, then by Proposition 3.2 we have $w \in A(u)$. Hence $z=L u+w+S(u) \in(\bar{G} \cap D(L))$.

THEOREM 4.2. Let $L+A+S \in \mathscr{F}_{X}(\mathrm{PM})$ (respectively, $L+A+S \in \mathscr{F}_{X}(\mathrm{QM})$ ) and assume that $S$ satisfies the two conditions:

(i) if $L u_{n}+w_{n}+S\left(u_{n}\right) \rightarrow h$ in $X^{*}$ with $w_{n} \in A\left(u_{n}\right)$, then the sequence $\left\{u_{n}\right\}$ is bounded in $X$,

(ii) there exists $R>0$ such that $\langle S(u), u\rangle>0$ for all $\|u\| \geq R$, Then $(L+A+$ $S)(D(L))=X^{*}$ (respectively, $(L+A+S)(D(L))$ is dense in $\left.X^{*}\right)$.

Proof. By condition (i) there exist two constants $R^{\prime} \geq R$ and $\delta>0$ such that

$$
\|L u+w+S(u)+\lambda J(u)-t h\| \geq \delta, \quad \text { where } w \in A(u),
$$

for all $u \in D(L),\|u\|=R^{\prime}, 0 \leq t \leq 1,0 \leq \lambda<\delta / R^{\prime}$. Thus we obtain

$$
D\left(L+A+S+\lambda J, B_{R}^{\prime}(X), h\right)=D\left(L+A+S+\lambda J, B_{R}^{\prime}(X), 0\right)
$$

whenever $0<\lambda<\delta / R^{\prime}$. Let

$$
F(t)(u)=L u+(1-t) J(u)+t(S(u)+A(u)+\lambda J(u)), \quad 0 \leq t \leq 1 .
$$

Since $(0,0) \in G(A)$, then by (ii), we obtain

$$
0 \notin F(t)(u)
$$

for all $t \in[0,1]$ and $0<\lambda<\delta / R^{\prime}$. Therefore, by invariance under homotopy, we have for all $0<\varepsilon<\delta / R^{\prime}$,

$$
D\left(L+A+S+\lambda J, B_{R}^{\prime}(X), 0\right)=D\left(L+J, B_{R}^{\prime}(X), 0\right)=1 .
$$

Hence there exists $u_{\lambda} \in D(L)$ such that $h-\lambda J\left(u_{\lambda}\right) \in L u_{\lambda}+A\left(u_{\lambda}\right)+S\left(u_{\lambda}\right)$. Letting $\lambda \rightarrow 0^{+}$, we have $h \in L u+A(u)+S(u)$ for some $u \in D(L)$ (Lemma 4.1) (respectively, $\left.\overline{(L+A+S)(D(L))}=X^{*}\right)$. 
5. Application to a parabolic variational inequality problem. Let $\Omega$ be a bounded open subset of $\mathbb{R}^{n}$ for some $n \geq 1$, and $Q$ the cylinder $\left.\Omega \times\right] 0, T$ [ for a given $T>0$, we consider the operators of the form

$$
\frac{\partial u}{\partial t}+A(u)+B(u)
$$

where

$$
B(u)=\sum_{|\alpha| \leq m}(-1)^{|\alpha|} D^{\alpha} A_{\alpha}\left(x, t, u, D u, \ldots, D^{m} u\right) .
$$

The supposed functions $A_{\alpha}$ satisfying the Carathéodory, polynomial growth conditions, and monotonicity such that the operator defined from $\mathscr{V}=L^{p}(0, T, V)$ with $V=W_{0}^{m, p}(\Omega)$ to $\mathscr{V}^{*}=L^{p^{\prime}}\left(0, T, V^{*}\right)$ by

$$
\langle S(u), v\rangle=\sum_{|\alpha| \leq m} \int_{Q} A_{\alpha}\left(x, t, u, D u, \ldots, D^{m} u\right) D^{\alpha} v, \quad u, v \in \mathscr{V}
$$

is bounded continuous pseudomonotone (see [4], for instance). Moreover, we assume that the functions $A_{\alpha}$ satisfy the coercivity condition. There exists $c>0$ and $k \in L^{1}(Q)$ such that

$$
\sum_{|\alpha| \leq m} A_{\alpha}(x, t, \xi) \xi_{\alpha} \geq c|\xi|^{p}-k(x, t)
$$

for all $(x, t) \in Q$ and $\xi \in \mathbb{R}^{N}$. Let $A$ be a bounded maximal monotone operator from $\mathscr{V}$ to $\mathscr{V}^{*}$. Indeed, let $\varphi$ be a convex lower semi-continuous function from a reflexive Banach space $X$ to $\mathbb{R} \cup\{+\infty\}$, then the subdifferential of $\varphi$ at $u$ in $X$, given by

$$
\partial \varphi(u)=\left\{w \in X^{*}: \varphi(v)-\varphi(u) \geq\langle w, v-u\rangle \forall v \in X\right\}
$$

is maximal monotone from $X$ to $2^{X^{*}}$. We assume in what follows that $2 \leq p<\infty$. Then, for each $u \in \mathscr{V}$ with $u^{\prime} \in \mathscr{V}^{*}$ which also belongs to $C\left([0, T], L^{2}(\Omega)\right)$, the initial condition $u(x, 0)=0$ in $\Omega$ makes sense. Thus the operator $\partial / \partial t$ induces a linear map from the subset $D(L)=\left\{v \in \mathscr{V} \mid v^{\prime} \in \mathscr{V}^{*}, v(0)=0\right\}$ of $\mathscr{V}$ to $\mathscr{V}^{*}$ by

$$
\langle L u, v\rangle=\int_{0}^{T}\left\langle u^{\prime}(t), v(t)\right\rangle d t, \quad u \in D(L), v \in \mathscr{V}
$$

here $u^{\prime}$ stands for the generalized derivative of $u$, that is,

$$
\int_{0}^{T} u^{\prime}(t) \varphi(t) d t=-\int_{0}^{T} u(t) \frac{\partial \varphi(t)}{\partial t} d t, \quad \forall \varphi \in C_{0}^{\infty}(0, T) .
$$

It can be shown (see [9]) that $L$ is a linear densely defined maximal monotone map.

THEOREM 5.1. Let $\Omega$ be an open bounded subset in $\mathbb{R}^{n}$ and $Q$ the cylinder $\left.\Omega \times\right] 0, T[$. Then the equation

$$
h \in L u+A(u)+S(u)
$$

admits a solution $u \in D(L)$. 
Proof. We use this result to give a new reformulation of a variational inequality problem-obstacle problem. We consider the operator $-\Delta_{p}$ defined from $L^{p}(0, T$, $\left.W_{0}^{1, p}(\Omega)\right)$ to $L^{p^{\prime}}\left(0, T, W^{-1, p^{\prime}}(\Omega)\right)$ by

$$
\left\langle-\Delta_{p}(u), v\right\rangle=\sum_{i=1}^{n} \int_{Q}|D u|^{p-2} \frac{\partial u}{\partial x_{i}} \frac{\partial v}{\partial x_{i}}, \quad u, v \in L^{p}\left(0, T, W_{0}^{1, p}(\Omega)\right) .
$$

It is a bounded continuous pseudomonotone map, then verifies the hypotheses assumed on the operator $B$ defined above. For $v \in L^{p}(Q)$, let

$$
v^{+}=\max (v, 0), \quad v^{-}=\min (v, 0) .
$$

We verify that

$$
\left\langle\Delta_{p}(u), u^{-}\right\rangle=\left\langle\Delta_{p}\left(u^{-}\right), u^{-}\right\rangle
$$

We define

$$
\begin{aligned}
& K=\left\{v \in W_{0}^{1, p}(\Omega): v \geq 0 \text { a.e. in } \Omega\right\}, \\
& \mathscr{K}=\left\{v \in L^{p}\left(0, T, W_{0}^{1, p}(\Omega)\right): v(t) \in K\right\} .
\end{aligned}
$$

The set $\mathscr{K}$ is a closed convex cone of $L^{p}\left(0, T, W_{0}^{1, p}(\Omega)\right)$. Let $h \in L^{p^{\prime}}(Q)$ be given. We are interested in the following problem:

$$
\left(P_{\mathscr{K}}\right)\left\{\begin{array}{l}
\text { find } u \in D(L) \cap \mathscr{K} \text { such that } \\
\langle L u, v-u\rangle+\left\langle-\Delta_{p}(u), v-u\right\rangle+(h, v-u) \geq 0, \quad \forall v \in \mathscr{K},
\end{array}\right.
$$

where $(\cdot, \cdot)$ designates the pairing between $L^{p}(Q)$ and $L^{p^{\prime}}(Q)$. The problem $\left(P_{\mathscr{K}}\right)$ is also called obstacle problem. If $\left(P_{\mathscr{K}}\right)$ admits a solution $u$, then it is unique. Indeed, we suppose that there exists two solutions $u_{1}$ and $u_{2}$, then from the inequality of $\left(P_{\mathscr{K}}\right)$ we obtain

$$
\begin{aligned}
& \left\langle L u_{1}, u_{2}-u_{1}\right\rangle+\left\langle-\Delta_{p}\left(u_{1}\right), u_{2}-u_{1}\right\rangle+\left(h, u_{2}-u_{1}\right) \geq 0 \\
& \left\langle L u_{2}, u_{1}-u_{2}\right\rangle+\left\langle-\Delta_{p}\left(u_{2}\right), u_{1}-u_{2}\right\rangle+\left(h, u_{1}-u_{2}\right) \geq 0
\end{aligned}
$$

and by adding (5.13), we obtain

$$
\left\langle L u_{1}-L u_{2}, u_{1}-u_{2}\right\rangle-\left\langle\Delta_{p}\left(u_{1}\right)-\Delta_{p}\left(u_{2}\right), u_{1}-u_{2}\right\rangle \leq 0 .
$$

Since $L$ is monotone and $-\Delta_{p}$ is strictly monotone, then we have $u_{1}=u_{2}$.

We consider the function $\varphi$ defined from $L^{p}\left(0, T, W_{0}^{1, p}(\Omega)\right)$ to $\mathbb{R}$ by $: v \mapsto \varphi(v)=$ $\left(h^{+}, v^{+}\right)$, it is continuous on $L^{p}\left(0, T, W_{0}^{1, p}(\Omega)\right)$ and convex. Hence the subdifferential of $\varphi: \partial \varphi$ is a maximal monotone operator from $L^{p}\left(0, T, W_{0}^{1, p}(\Omega)\right)$ to $L^{p^{\prime}}\left(0, T, W^{-1, p^{\prime}}(\Omega)\right)$ (see $[7,9])$. Furthermore, we verify that $\partial \varphi$ is bounded.

THEOREM 5.2. The problem

$$
(P)\left\{\begin{array}{r}
\text { find } u \in D(L) \text { such that } \\
\langle L u, v-u\rangle+\left\langle-\Delta_{p}(u), v-u\right\rangle+\varphi(v)-\varphi(u)+\left(h^{-}, v-u\right) \geq 0, \\
\forall v \in L^{p}\left(0, T, W_{0}^{1, p}(\Omega)\right)
\end{array}\right.
$$

admits a unique solution and it is equivalent to problem $\left(P_{\mathscr{K}}\right)$. 
Proof. The problem $(P)$ is equivalent to find $u \in D(L)$ such that

$$
h^{-} \in L u-\Delta_{p}(u)+\partial \varphi(u) \text {. }
$$

Then by Theorem 5.1 and the strict monotonicity of $-\Delta_{p}$, we conclude that $(P)$ admits a unique solution. Let $u$ be this solution. It suffices to show that $u$ is an element of $\mathscr{K}$ to complete the proof. For $v=u^{+} \in \mathscr{K}$, the inequality of $(P)$ becomes

$$
\left\langle L u,-u^{-}\right\rangle+\left\langle-\Delta_{p}(u),-u^{-}\right\rangle+\left(h^{-},-u^{-}\right) \geq 0 .
$$

Since $\left\langle L u,-u^{-}\right\rangle \leq 0$ (see [8]) and $\left(h^{-},-u^{-}\right) \leq 0$, we have

$$
\left\langle-\Delta_{p}(u), u^{-}\right\rangle \leq 0 \text {. }
$$

Then by (5.11), we obtain

$$
\left\langle-\Delta_{p}\left(u^{-}\right), u^{-}\right\rangle \leq 0 .
$$

Hence $\left\|u^{-}\right\|_{L^{p}\left(0, T, W_{0}^{1, p}(\Omega)\right)}=0$, therefore $u \in \mathscr{K}$.

\section{REFERENCES}

[1] H. Amann and S. A. Weiss, On the uniqueness of the topological degree, Math. Z. 130 (1973), 39-54. MR 49\#11326. Zbl 249.55004.

[2] H. Attouch, Variational Convergence for Functions and Operators, Applicable Mathematics Series, Pitman (Advanced Publishing Program), Boston, Mass., 1984. MR 86f:49002. Zbl 561.49012.

[3] J. Berkovits and V. Mustonen, On the topological degree for mappings of monotone type, Nonlinear Anal. 10 (1986), no. 12, 1373-1383. MR 88b:47073. Zbl 605.47060.

[4] - Topological degree for perturbations of linear maximal monotone mappings and applications to a class of parabolic problems, Rend. Mat. Appl. (7) 12 (1992), no. 3, 597-621. MR 94f:47073. Zbl 806.47055.

[5] F. E. Browder, Fixed point theory and nonlinear problems, Bull. Amer. Math. Soc. (N.S.) 9 (1983), no. 1, 1-39. MR 84h:58027. Zbl 533.47053.

[6]__ Degree theory for nonlinear mappings, Nonlinear Functional Analysis and its Applications, Part 1 (Berkeley, Calif., 1983), Proc. Sympos. Pure Math., Part 1, vol. 45, Amer. Math. Soc., Providence, R.I., 1986, pp. 203-226. MR 87g:47108. Zbl 601.47050.

[7] K. Deimling, Nonlinear Functional Analysis, Springer-Verlag, Berlin, New york, 1985. MR 86j:47001. Zbl 559.47040.

[8] J.-L. Lions, Quelques Méthodes de Résolution des Problèmes aux Limites Non Linéaires, Dunod; Gauthier-Villars, Paris, 1969 (French). MR 41\#4326. Zbl 189.40603.

[9] E. Zeidler, Nonlinear Functional Analysis and its Applications. II/B, Nonlinear monotone operators. Translated from the German by the author and Leo F. Boron, SpringerVerlag, New York, Berlin, Heidelberg, 1990. MR 91b:47002. Zbl 684.47029.

A. Addou: University Mohammed I, FaCulty of SCIENCES, DePaRtMent of MATHEMATics AND COMPUTING, OUJDA, MOROCCO

E-mail address: addou@sciences. univ-oujda.ac.ma

B. Mermri: UniVERSITY MOHAMmed I, FACUlTy of SCIENCES, DEPARTMENT OF MATHEMATICS AND COMPUTING, OUJDA, MOROCCO

E-mail address: mermri@sciences . univ-oujda.ac.ma 


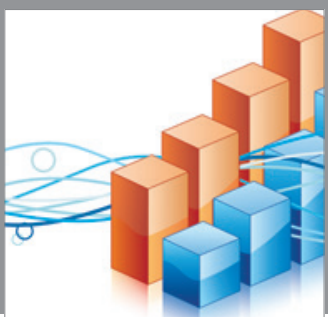

Advances in

Operations Research

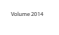

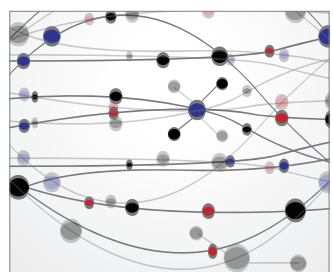

\section{The Scientific} World Journal
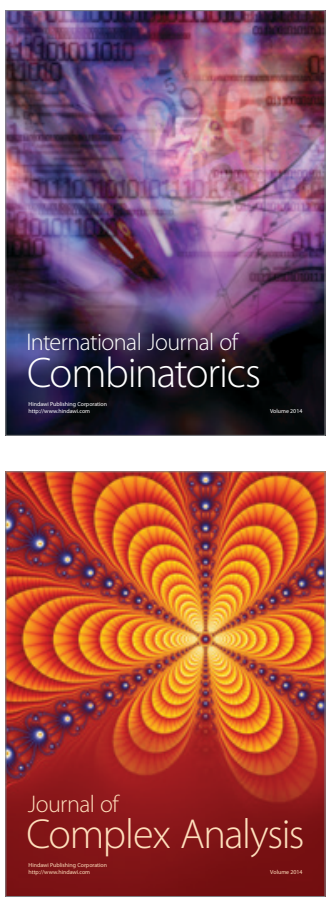

International Journal of

Mathematics and

Mathematical

Sciences
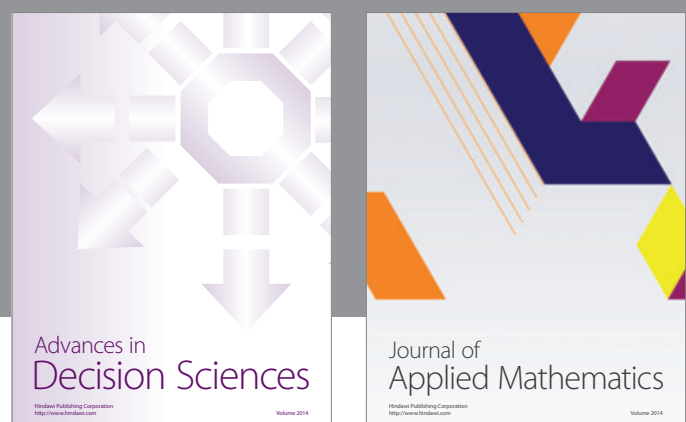

Journal of

Applied Mathematics
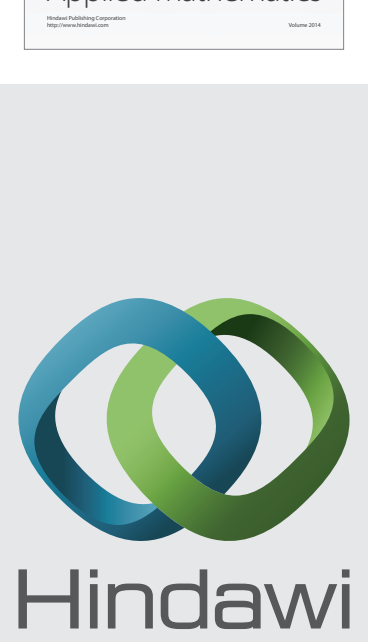

Submit your manuscripts at http://www.hindawi.com
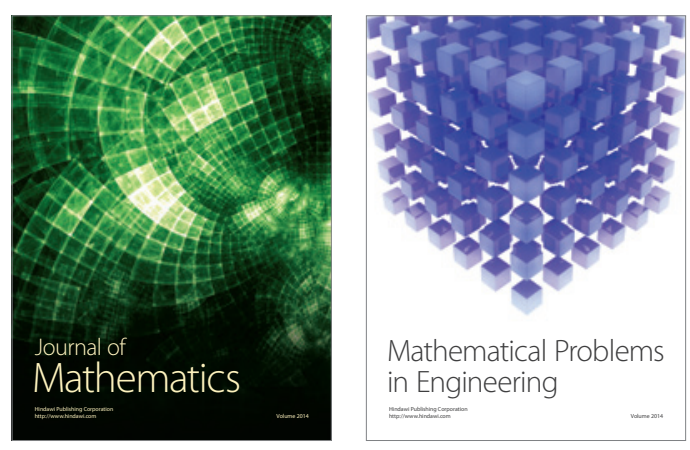

Mathematical Problems in Engineering
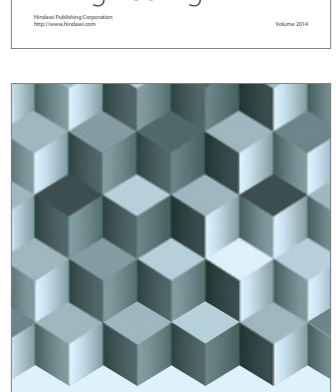

Journal of

Function Spaces
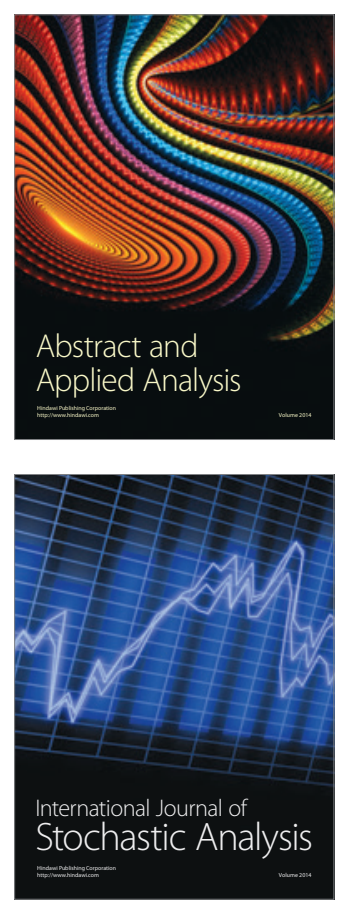

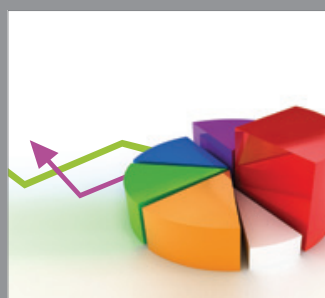

ournal of

Probability and Statistics

Promensencen
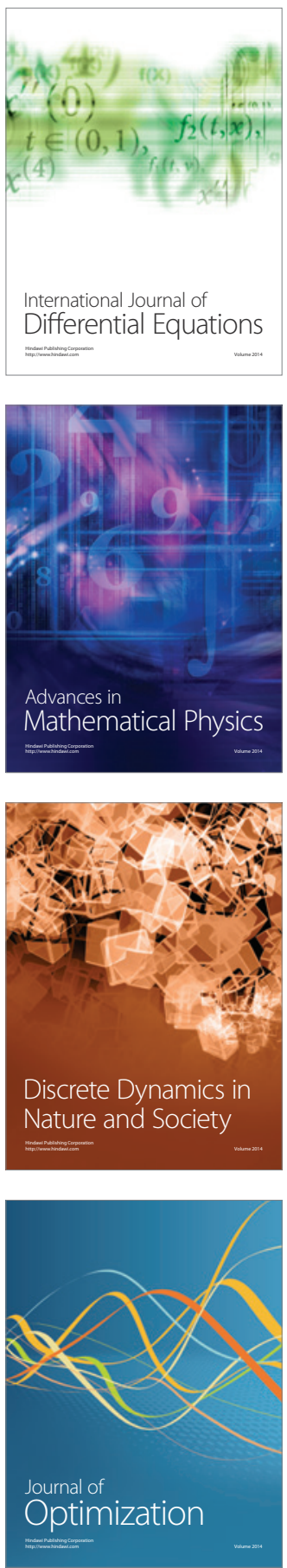